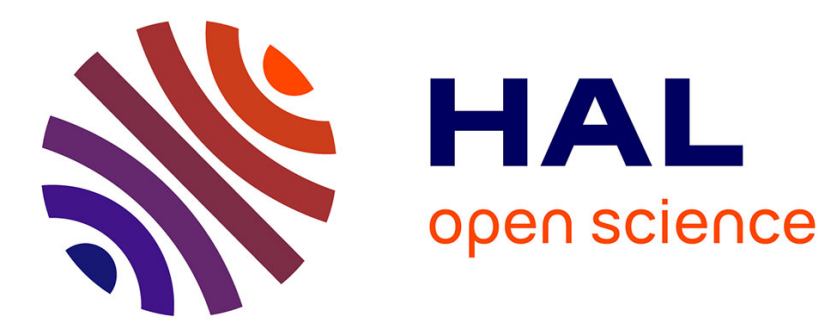

\title{
L'utilisation de la figure de Crésus dans l'idéologie aristocratique athénienne: Solon, Alcméon, Miltiade et le dernier roi de Lydie
}

Alain Duplouy

\section{To cite this version:}

Alain Duplouy. L'utilisation de la figure de Crésus dans l'idéologie aristocratique athénienne: Solon, Alcméon, Miltiade et le dernier roi de Lydie. L'Antiquité classique, 1999, 68, pp.1-22. hal-02404529

HAL Id: hal-02404529

https: / hal-paris1.archives-ouvertes.fr/hal-02404529

Submitted on 11 Dec 2019

HAL is a multi-disciplinary open access archive for the deposit and dissemination of scientific research documents, whether they are published or not. The documents may come from teaching and research institutions in France or abroad, or from public or private research centers.
L'archive ouverte pluridisciplinaire HAL, est destinée au dépôt et à la diffusion de documents scientifiques de niveau recherche, publiés ou non, émanant des établissements d'enseignement et de recherche français ou étrangers, des laboratoires publics ou privés. 


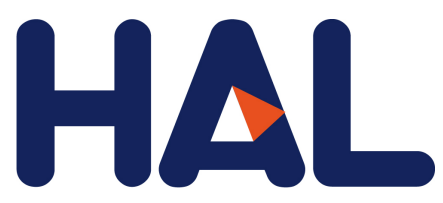

archives-ouvertes

\section{L'utilisation de la figure de Crésus dans l'idéologie aristocratique athénienne: Solon, Alcméon, Miltiade et le dernier roi de Lydie}

Alain Duplouy

\section{- To cite this version:}

Alain Duplouy. L'utilisation de la figure de Crésus dans l'idéologie aristocratique athénienne: Solon, Alcméon, Miltiade et le dernier roi de Lydie. L'Antiquité classique, Association L'Antiquité classique, 1999, 68, pp.1-22. hal-02404529

HAL Id: hal-02404529

https://hal-paris1.archives-ouvertes.fr/hal-02404529

Submitted on 11 Dec 2019

HAL is a multi-disciplinary open access archive for the deposit and dissemination of scientific research documents, whether they are published or not. The documents may come from teaching and research institutions in France or abroad, or from public or private research centers.
L'archive ouverte pluridisciplinaire HAL, est destinée au dépôt et à la diffusion de documents scientifiques de niveau recherche, publiés ou non, émanant des établissements d'enseignement et de recherche français ou étrangers, des laboratoires publics ou privés. 


\title{
L'utilisation de la figure de Crésus dans l'idéologie aristocratique athénienne
}

\author{
Solon, Alcméon, Miltiade et le dernier roi de Lydie*
}

Les Histoires d'Hérodote contiennent trois récits qui évoquent les contacts privilégiés qu'aurait entretenus le dernier roi de Lydie, Crésus, avec les Athéniens Solon, Alcméon et Miltiade l'Ancien. La discussion, plusieurs fois reprise par les auteurs postérieurs, entre Solon et Crésus à propos du «bonheur» (I, 29-33) appartient au livre I et à ce qu'il est convenu d'appeler le logos de Crésus, tandis qu'il faut attendre le livre VI et le récit de la première guerre médique pour rencontrer à nouveau le Lydien et l'évocation de ses relations avec Alcméon (VI, 125) et Miltiade l'Ancien (VI, 37). Loin d'être un élément insignifiant, l'emplacement de ces épisodes au sein de l'œuvre d'Hérodote conditionna en fait, dans une large mesure, la bibliographie contemporaine : bien qu'il y fût également question du roi lydien, les exégètes traditionnels du logos de Crésus ${ }^{1}$ se préoccupèrent peu ou pas du livre VI, laissant ce soin aux historiens de l'Athènes archaïque, qui eux, en revanche, ne s'intéressaient pas directement au personnage de Crésus ni à un dialogue cherchant à déterminer «le plus heureux des hommes». L'exégèse des deux anecdotes du livre VI n'a donc jamais véritablement concerné le dernier roi de Lydie, auquel est restée attachée une vision largement préconçue du monarque oriental. À travers un réexamen de l'ensemble du volet athénien du dossier «Crésus», on s'attachera donc particulièrement à l'image que les sources classiques ont conservée d'un personnage pour lequel nous ne disposons, à l'exception de la dédicace des colonnes de l'Artémision $d^{\prime}$ Ephèse $^{2}$, d'aucune source écrite contemporaine. Afin de relancer

\footnotetext{
* Je tiens à exprimer ici toute ma reconnaissance envers le professeur Didier Viviers, qui fut quelque part à l'origine de cette étude et qui a aimablement accepté d'en relire le manuscrit.

Fr. HellmanN, Herodots Kroisos-Logos, Berlin, 1934 [réédité largement par une traduction italienne partielle: L. BELloni, L'evento di Creso : il racconto di Erodoto secondo Hellmann, dans Aevum Antiquum, 9 (1996), p. 11-48]; A. HEUss, Motive von Herodots lydischem Logos, dans Hermes, 101 (1973), p. 385-419; A.C. SHEFFIELD, Herodotus' Portrait of Croesus. A Study in Historical Artistry, Diss. Standfort, 1973; H. ERBSE, Studien zum Verständnis Herodots, Berlin - New York, 1992, p. 10-30.

2 Hérodote (I, 92) déclare que Crésus a offert «la plupart des colonnes», ce qui fut confirmé par la découverte de la dédicace en grec (LonDres, Brit. Mus. B 16 et B 32; I.K. XV, 1518) et vraisemblablement en lydien (LONDRES, Brit. Mus. B 136).
} 
l'intérêt de l'histoire de la Lydie archaïque, il nous faut en effet commencer par comprendre la place qu'occupait encore, dans la Grèce classique, un personnage mort au milieu du VIe siècle.

L'histoire de Solon à Sardes est célèbre; rappelons-en cependant les traits essentiels. Lorsque l'Athénien se présenta à la cour du roi lydien, Crésus lui demanda de nommer le «plus heureux des hommes» (ỏ $\lambda \beta ı \omega \tau \tau \tau \tau)$ ), pensant, grâce à sa richesse, avoir droit à ce titre; mais Solon lui préféra l'Athénien Tellos et les Argiens Cléobis et Biton, suscitant ainsi la colère du roi, qui le congédia. D'après Hérodote, Solon vint à Sardes au cours des dix années qui suivirent la promulgation de sa législation, alors que Crésus était au faîte de sa puissance (I, 29-30). La rencontre de Solon avec le roi mermnade pose bien évidemment un problème d'authenticité, puisque l'archontat de l'Athénien est fixé avec précision à l'année 594/33 , alors que Crésus ne régna pas avant la fin des années $560^{4}$. Il est dès lors impossible que Solon ait rencontré Crésus au cours de son apodèmia. Plutarque (Solon, 27,1) témoignait déjà des doutes que certains émettaient sur cet épisode en raison précisément de l'invraisemblance chronologique, choisissant, quant à lui, de croire en la véracité de ce récit si célèbre et si conforme au caractère de Solon. C'est à juste titre que la recherche actuelle a, en grande majorité, rejeté cette entrevue comme n'étant pas historique et a mis en évidence par la même occasion la nature bien particulière du discours 5 .

3 DIOGÈnE LAËrCE I, 62 (qui précise que c'est lors de son archontat que Solon fit passer ses lois); ARISTOTE, Const. Ath., 14, 1. - Sur la correction du texte d'Aristote, voir p. ex. T.J. CADoux, The Athenian Archons from Kreon to Hypsichides, dans JHS, 68 (1948), p. 70-123, partic. p. 93-99.

4 La chronologie du règne de Crésus est directement liée à celle de la prise de Sardes, qui repose sur le F 28 d'Apollodore (FGrHist 244), pour lequel H. KaLETSCH, Zur lydischen Chronologie, dans Historia, 7 (1958), p. 1-47, partic. p. 39-46 et A.A. Mosshammer, The Chronicle of Eusebius and Greek Chronographic Tradition, Lewisburg, 1979, p. 255262 et p. 346 , n. 6 et 7 , substituèrent à la date traditionnelle de 546/5 celle de 547/6. La durée de règne de Crésus est fixée à 15 ans par Eusèbe, mais également par le Marbre de Paros (ce qui permet de remonter cette donnée au III $^{\mathrm{e}}$ siècle av. J.-C). Seul Hérodote (I, 86) lui en attribue 14.

$5 \quad \mathrm{Au}$ prix d'incroyables détours, quelques-uns ont cependant vainement tenté de recomposer complètement l'histoire athénienne de la première moitié du $\mathrm{VI}^{\mathrm{e}}$ siècle, tantôt en contestant la validité de la date archontale, tantôt en distinguant l'archontat de la législation. Deux auteurs, en particulier, furent d'ardents défenseurs de la vraisemblance chronologique de cette rencontre : M. Miller, The Herodotean Croesus, dans Klio, 41 (1963), p. 58-94; EAD., The Accepted Date for Solon: Precise but Wrong?, dans Arethusa, 2 (1969), p. 62-86; S.S. MARKIANOS, The Chronology of the Herodotean Solon, dans

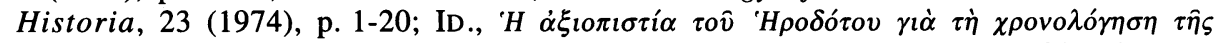

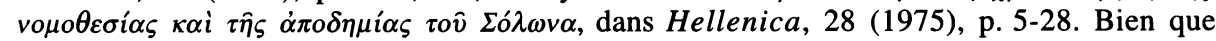
leurs positions fussent récemment réaffirmées par W. LAPINI, Il POxy. 664 di Eraclide Pontico e la cronologia dei Cipselidi, Florence, 1996, p. 41-57, leurs arguments princi- 
L'épisode a déjà fait l'objet de nombreuses études et il pourrait paraître inutile d'ajouter une référence supplémentaire à une bibliographie déjà longue ${ }^{6}$. Pourtant, dans ce dialogue, seuls les propos de Solon ont fait l'objet d'un examen minutieux, alors que le personnage de Crésus a finalement peu retenu l'attention des chercheurs, son rôle paraissant évident. La clé d'interprétation unanime de l'épisode fut en effet d'envisager le désaccord qui se dégage de la discussion comme le témoin d'une opposition radicale entre la sagesse grecque et l'ignorance barbare, entre le citoyen et le monarque oriental. Dès 1930, Otto Regenbogen, dans un article qui demeure l'analyse de référence, écrivait à propos de Tellos l'Athénien : Die Tellosgeschichte [...] setzt den echten Hellenen gegen den Barbaren und verherrlicht gegenüber orientalischer Pracht und asiatischem Reichtum die wahren Lebensgüter des hellenischen Mannes, d.h. des hellenischen Bürgers ${ }^{7}$. Cette position doit cependant être nuancée, car, si la discussion entre Solon et Crésus débouche effectivement sur un désaccord majeur entre les deux hommes, celui-ci ne semble nullement relever d'une opposition du type Grec - Barbare.

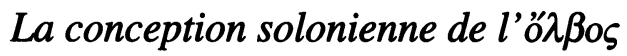

À travers une argumentation confuse et souvent répétitive, l'Athénien est amené à définir deux types bien distincts de «bonheur»:

paux avaient été contestés par R.W. WALlACE, The Date of Solon's Reforms, dans AJAH, 8 (1983), p. 81-95, et il n'y a pas lieu de revenir sur cette question.

6 Sans aucunement prétendre à l'exhaustivité, on retiendra, en plus des références citées à la note 1 : L. WeBER, Tellos, Kleobis und Biton, dans Philologus, 82 (1927), p. 154-166; O. Regenbogen, Die Geschichte von Solon und Krösus, dans Gymnasium, 41 (1930), p. 1-20 [reproduit dans W. MARG, Herodot. Eine Auswahl aus der neueren Forschung, Darmstadt, $1965^{2}$, p. 375-403]; K. NAWRATIL, Solon bei Herodot, dans WS, 60 (1942), p. 1-8; J. DeFrADAS, Les themes de la propagande delphique, Paris, 1954, p. 217-223; T. KRISCHER, Solon und Kroisos, dans WS, 77 (1964), p. 174-177; H.R. IMMERWAHR, Form and Thought in Herodotus, Cleveland, 1966, p. 154-161; K. VON FRITZ, Die griechische Geschichtsschreibung, Berlin, 1967, t. I, p. 216-223; P. OLIVA, Die Geschichte von Kroisos und Solon, dans Das Altertum, 21 (1975), p. 175-191; H.-P. STAHL, Learning through Suffering? Croesus' Conversations in the History of Herodotus, dans YClS, 24 (1975), p. 1-36; C.C. ChIAsson, The Herodotean Solon, dans GRBS, 27 (1986), p. 249-262; M. LloyD, Cleobis and Biton (Herodotus 1,31), dans Hermes, 115 (1987), p. 22-28; P. Oliva, Solon. Legende und Wirklichkeit, Constance, 1988, p. 1117; Tr.S. BROWn, Solon and Croesus (Hdt 1.29), dans AHB, 3 (1989), p. 1-4; S.O. SHAPIRO, Herodotus and Solon, dans ClAnt, 15 (1996), p. 348-364.

7 O. Regenbogen, loc. cit. (n. 6), p. 381-382. - Citons également deux études récentes, la première sur Solon, la seconde sur Hérodote. P. OlivA, loc. cit. (n. 6) déclarait Die Morale der Geschichte ist offenkundig. Die Erfahrung und Besonnenheit des griechischen Weisen werden dem Hochmut und der Verblendung des barbarischen Herrschers gegenübergestellt (p. 14), tandis que H. ERBSE, op. cit. (n. 1) écrivait In Kroisos' Gespräch mit Solon (1,29-33) tritt die griechische (attische) Sophrosyne der orientalischen Vermessenheit gegenüber und enthüllt deren Unsicherheit, ja Hohlheit (p. 12). 


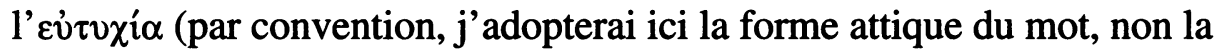
forme ionienne employée par Hérodote) et l'ö $\lambda \beta$ os. C'est parce qu'aucune langue moderne ne peut rendre avec précision la définition exacte de ces deux termes ${ }^{8}$, qu'il faut $s^{\prime}$ attacher à la signification très précise que leur accordait Solon.

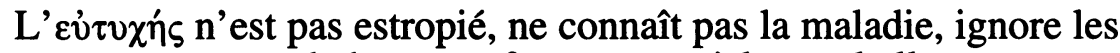
maux en tout genre, a de beaux enfants et possède une belle apparence

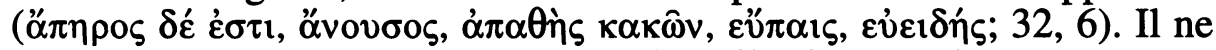
s'agit pas pour Solon de critères absolus : il sait qu'un homme ne peut tous les réunir, car «s'il possède l'un, il lui manque l'autre» $(32,8)$. Qui plus est, la vie est longue - il fixe sa durée à 70 ans soit, prend-t-il soin de préciser, 26.250 jours -, et chaque jour est susceptible d'apporter l'un ou l'autre malheur ( $\sigma \nu \mu \varphi \circ \rho \eta \dot{;} ; 32,5)$. Enfin, la divinité est jalouse et

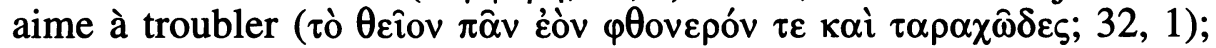
elle pourrait totalement renverser une situation qui semblait favorable

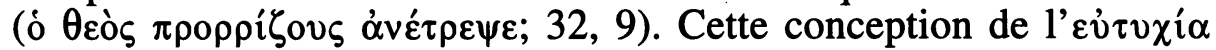
comme un bonheur provisoire, soumis à la bonne volonté des dieux, n'est pas propre à ce passage d'Hérodote. Son œuvre en recèle maintes occurrences ${ }^{9}$. C'est, au demeurant, un sentiment partagé par de nombreux auteurs grecs et on pourrait sans aucune difficulté multiplier les exemples ${ }^{10}$.

En revanche, le terme ö $\lambda \beta$ ıo est excessivement rare chez Hérodote et il ne se rencontre pratiquement qu'en association avec Solon ${ }^{11}$.

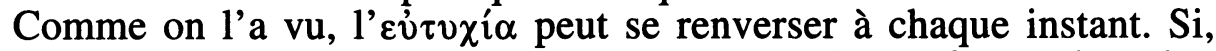
malgré tout, quelqu'un parvient à conserver cette bonne fortune jusqu'au

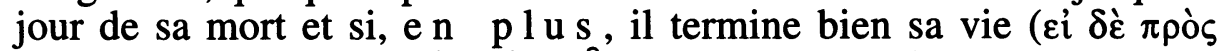

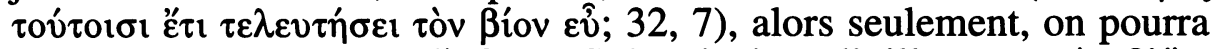
qualifier cet homme d'ő $\lambda \beta$ ıьos. Solon insiste d'ailleurs : $\pi \rho i v \delta^{\prime} \grave{\alpha}^{2}$

8 Leur traduction uniforme par «bonheur», «Glück» ou «happiness» ne permet pas toujours de rendre la subtilité des propos de Solon. On ne peut pas davantage admettre la

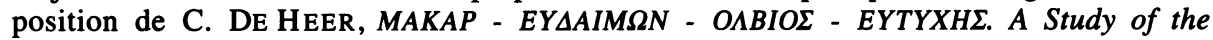
Semantic Field Denoting Happiness in Ancient Greek to the End of the 5th Century B.C.,

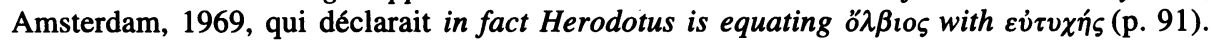
De même, en dépit d'idées intéressantes, T. KRISCHER, Drei Definitionen des Glücks. Pindar, Herodot, Prodikos, dans RhM, 136 (1993), p. 213-222, passe insensiblement de

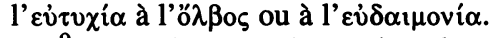

9 La lettre qu'Amasis adresse à Polycrate (III, 39-43) est le parallèle le plus fréquemment cité dans la bibliographie, mais il est loin d'être le seul. Il suffit en effet de se

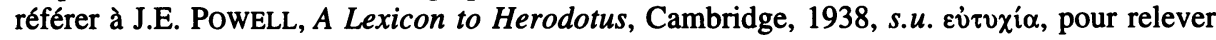
d'autres exemples.

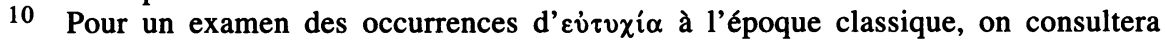
l'ouvrage commode de C. DE HEER, op. cit. (n. 8), p. 72-81.

11 J.E. PowELL, op. cit. (n. 9) a relevé 22 occurrences du terme ö $\lambda \beta$ o̧ et de ses dérivés (ő $\lambda \beta ı \varsigma, \alpha \dot{\alpha} v o ́ \lambda \beta o \varsigma, \alpha \dot{\alpha} \lambda_{\lambda \beta} \beta_{10 \varsigma}$ ). Parmi celles-ci, seules cinq se rencontrent sans être liées au personnage de Solon (I, 85, 216; VI, 24, 61; VIII, 75). 


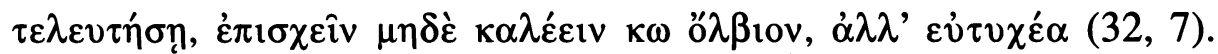
Une belle mort apparaît donc comme l'élément fondamental dans

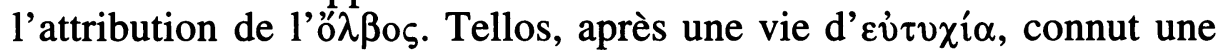
mort qualifiée de $\lambda \alpha \mu \pi \rho \circ \alpha \alpha \tau \eta ~(30,4)$; celle de Cléobis et Biton est dite

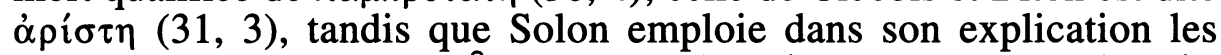

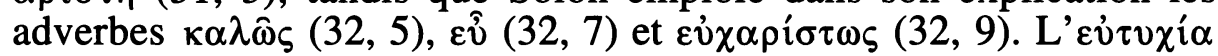
peut donc éventuellement conduire à l'ó $\lambda \beta 0 \varsigma$, mais, en aucun cas, un homme encore vivant ne peut prétendre à ce titre, comme le faisait Crésus. C'est sans doute là le point essentiel du désaccord entre les deux hommes ${ }^{12}$.

\section{L'öגßos selon Crésus : un concept aristocratique}

Alors que, dans l'esprit de Solon, l'ő $\lambda \beta$ $_{\text {c }}$ demeure inaccessible à l'homme tant qu'il vit, Crésus aspirait clairement au statut d'ó $\lambda \beta 1 \omega ́ \tau \alpha \tau o \zeta$. Gageons donc que le roi lydien se référait à une autre conception de l'ő $\lambda \beta$ os, une conception qui n'entretenait aucun rapport avec la mort. Comme l'avait très justement noté $C$. De Heer, le sens du mot diffère fondamentalement selon qu'Hérodote le place dans la bouche de Solon ou dans celle de Crésus, mais fallait-il pour autant parler de misunderstanding between Oriental and Greek ${ }^{13}$ ?

Avant de poser la question fatidique à Solon, Crésus prend soin, afin d'orienter sa réponse, de le promener parmi ses trésors et de lui montrer la grandeur de son palais (

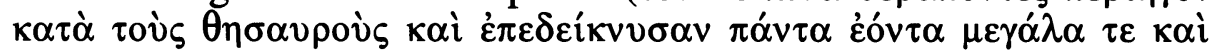
ő $\lambda \beta \mathrm{i} \alpha ; 30,1)$. Tel qu'Hérodote l'utilise ici pour décrire la situation de Crésus, l'adjectif ő $\lambda \beta$ ıos s'applique manifestement à la richesse et au mode de vie somptueux du roi lydien; cette interprétation se confirme

12 Bien sûr, la nature précise de l'ő $\lambda \beta \wp_{\varsigma}$ solonien, les nuances qui existent entre

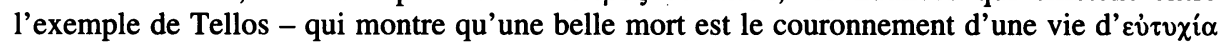

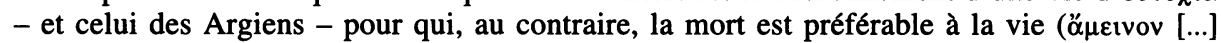

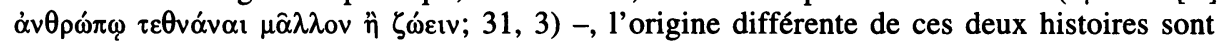
autant de questions qui ont lieu d'être posées, mais qui ont déjà été discutées de nombreuses fois [voir en dernier lieu M. LLOYD, loc. cit. (n. 6)]. Je me contenterai de noter que la

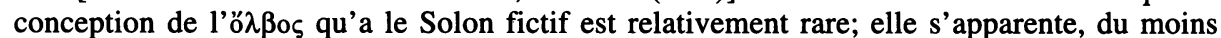
pour l'histoire de Cléobis et Biton, à une conception post mortem, vraisemblablement influencée par les idées delphiques, telles qu'on les connaît par exemple à travers l'histoire de Trophonios et Agamédès [voir déjà O. Regenbogen, loc. cit. (n. 6), p. 383-389]. Ch. PICARD, Déméter et les jumeaux d'Argos, dans RHR, 96 (1927), p. 365-398 et L.-M. L'HOMME-WÉRY, Solon, libérateur d'Éleusis dans les «Histoires» d'Hérodote, dans REG, 107 (1994), p. 362-380 ont, quant à eux, défendu l'idée d'une origine éleusinienne de l'histoire des Argiens. Sur la place d'Éleusis dans l'action du personnage historique de Solon, voir dernièrement L.-M. L'HOMME-WÉRY, La perspective éleusinienne dans la politique de Solon, Genève, 1996.

13 C. DE HEER, op. cit. (n. 8), p. 71. 
d'ailleurs avec la troisième et dernière partie du discours de Solon (32). Après avoir exposé les exemples concrets de Tellos (30) et des Argiens (31), l'Athénien se sent effectivement obligé de préciser sa pensée en expliquant la place qu'occupent les biens matériels dans sa conception de

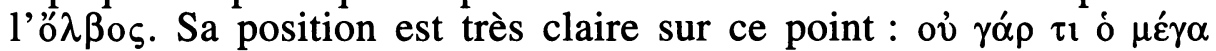

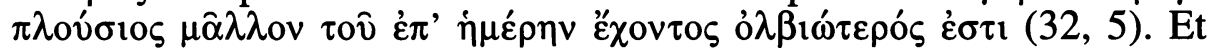
d'ajouter qu'il existe à la fois des gens très riches dont on dira par la suite qu'ils étaient $\alpha$ $v o ́ \lambda \beta 10$, et des gens qui vivent modestement tout en étant $\varepsilon \dot{\tau} \tau \cup \chi \varepsilon ́ \varepsilon \varsigma(32,5-6)^{14}$. En se livrant à une telle relativisation des biens matériels ${ }^{15}$, Solon nous offre ainsi la confirmation que, dans l'esprit de Crésus, l'ő $\lambda \beta \beta_{\text {o }}$ s'identifiait bel et bien à la possession d'une fortune matérielle ${ }^{16}$.

Le dialogue entre Solon et Crésus ne pouvait donc déboucher que sur une aporia, sur une incompréhension totale entre deux hommes qui se référaient à deux conceptions totalement incompatibles de l'ő $\lambda ß o s$. La question qu'il faut maintenant se poser est de savoir si la conception de Crésus s'apparente effectivement à une vision barbare du bonheur et s'oppose ainsi à la sagesse toute grecque d'un Solon. Force est alors de constater que le sens qu'accorde Crésus à ce terme correspond en fait à son champ sémantique le plus traditionnel dans les textes grecs archaïques et classiques ${ }^{17}$. Dès Homère, le mot évoque essentiellement un bonheur matériel, fait de la possession d'une maison et de grandes

14 On notera la cohérence parfaite de l'emploi des deux termes chez Hérodote. Même si le riche est capable de supporter les malheurs qui s'abattent sur lui grâce à son argent, par le fait même qu'il connaisse ces malheurs, il ne pourra être considéré que comme óvó $\lambda \beta$ ıos. D'autre part, celui qui vit modestement ne peut bénéficier directement du titre d'ő $\lambda \beta ı \varsigma$, puisque son $\varepsilon \dot{\tau} \tau \chi \chi i \alpha$ n'est pas nécessairement définitive, même si elle lui permet d'écarter bon nombre de malheurs.

15 Il ne s'agit en effet pas, dans l'optique de Solon, d'une condamnation sans appel des biens matériels. Tellos réussit sa vie «comme cela se peut chez nous» ( $\dot{\omega} \varsigma \tau \grave{\alpha} \pi \alpha \rho$ ' $\dot{\eta} \mu i ̂ v$; $30,4)$ et les Argiens disposent de ressources suffisantes $(31,2)$. Solon précise également que l'homme riche peut subvenir à ses désirs et supporter ses peines plus facilement qu'un autre $(32,6)$. - Ce ne sera pas toujours le cas chez ceux qui, après Hérodote, raconteront encore cette même histoire. En comparant le récit de l'historien d'Halicarnasse à celui de Diodore (IX, 27), Anna Santoni a en effet mis en évidence la valeur différente que les deux auteurs accordaient à la notion de «richesse». Contrairement au Solon hérodotéen, le personnage de Diodore rejette complètement tout bien matériel comme immoral, témoignant d'une influence récente de la philosophie cynique sur l'épisode. $C f$. A. SANTONI, Temi e motivi di interesse socio-economico nella leggenda dei 'Sette Sapienti', dans ASNP, ser. III, 13 (1983), p. 91-160, partic. p. 136-138.

16 T. KRISCHER, loc. cit. (n. 8), p. 220 arrivait à une conclusion semblable : Entsprechend ist das Wort öגßıৎ aus dem Munde des Kroisos voll verständlich; gemeint ist ein Mensch, der mit Glückgütern gesegnet ist.

17 Davantage d'exemples sont donnés dans les études de C. DE HEER, op. cit. (n. 8), p. $8,12-15$ (Homère), p. 32-38 (poésie archaïque), p. 67-72 (époque classique) et de P. LÉVÊQUE, "Oגßros et la félicité des initiés, dans L. HADERMANN-MISGUICH et G. RAEPSAET (éd.), Rayonnement grec. Hommages à Charles Delvoye, Bruxelles, 1982, p. 113-126. 
richesses, auquel s'ajoutent la présence d'une épouse et d'enfants ainsi que le pouvoir de commander. Chez les poètes lyriques, le concept s'enrichit d'une notion de plaisir et l'amour apparaît comme générateur d'ő $\lambda \beta o s$, tant dans le mariage que dans l'amour pédérastique. Je citerai les vers de Solon, qui reflètent l'idéal d'une classe et un mode de vie

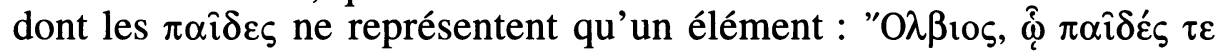

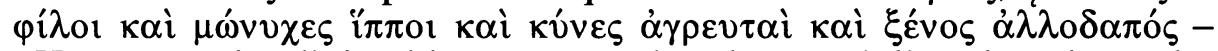
«Heureux qui a d'aimables garçons, des chevaux à l'ongle unique, des chiens de chasse et un hôte étranger» ${ }^{18}$. Le mot ő $\lambda \beta \sigma_{\zeta}$ est utilisé par Pindare et Bacchylide pour désigner le bonheur des athlètes victorieux. On rappellera, notamment, l'emploi de l'adjectif dans la III' Épinicie de Bacchylide, lorsque le poète chante la victoire de Hiéron de Syracuse

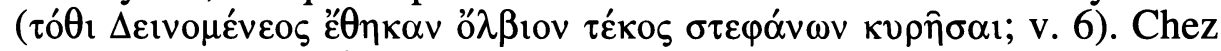
les Tragiques, les ő $\lambda \beta$ ıo sont principalement les riches et les nobles, ceux qui disposent de biens matériels et d'un statut social élevé. Un vers

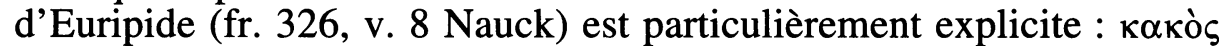

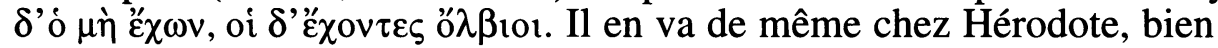
que le mot soit très rare en dehors du dialogue mettant en scène Solon et Crésus. L'historien raconte comment Thémistocle avait rendu ő $\lambda \beta 10 \varsigma$ son serviteur Sikinnos au moyen de $\chi \rho \eta \dot{\mu} \mu \alpha \sigma$, lorsqu'il en avait fait un citoyen de Thespies (VIII, 75). Toutes ces occurrences définissent un bonheur de classe et l'ő $\lambda \beta$ o de Crésus apparaît essentiellement comme un concept aristocratique. Le terme se rencontre tant dans les chants d'hyménée que dans les odes olympiques; il évoque aussi bien la richesse matérielle que l'amour pédérastique ou la gloire des vainqueurs. L'étendue du champ sémantique ainsi couvert correspond en fait au mode de vie des individus auxquels il s'appliquait. Le roi lydien luimême adopte un comportement sans équivoque, témoignant d'une fierté extraordinaire de sa condition. Une fois que Solon a cité les noms de Tellos, Cléobis et Biton, il s'irrite et demande : «notre bonheur est-il à ce point mis de côté et réduit à néant, que tu ne nous rendes même pas

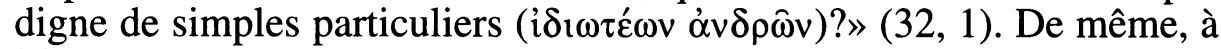
l'issue de l'entrevue et en dépit des explications précises de Solon sur le statut de la richesse, Crésus congédie l'Athénien, considérant ses propos comme insensés $(\alpha \dot{\alpha} \mu \alpha \theta \dot{\varepsilon} \alpha, 33)$.

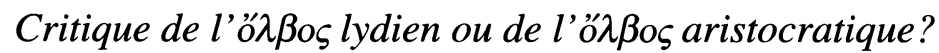

On le voit, Crésus, par l'usage qu'il fait du terme ő $\lambda \beta$ os, s'identifie tout à fait à un aristocrate grec; car si Solon combat

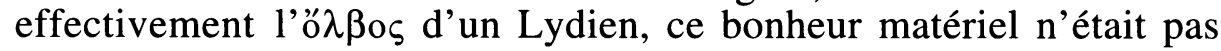

18 Solon, fr. 17 Gentili-Prato (Leipzig, 1979). 
envisagé autrement par les grands seigneurs grecs. Cette position de Crésus vis-à-vis de l'ổ $\lambda$ os n'étant pas spécifiquement lydienne, comme le laissaient supposer les commentateurs depuis Regenbogen, on est finalement amené à se demander si la «nationalité» de Crésus joue réellement un rôle dans ce récit. En d'autres termes, Solon critique-t-il l'attitude d'un Barbare, davantage que celle d'un aristocrate qui fait preuve d'ü $\beta$ pı en se croyant «heureux» (ö $\lambda \beta 10 \varsigma$ ) parce que riche ? La réponse tient, je pense, à la nature même de cet épisode.

Comme on l'a souvent montré, cette histoire s'est élaborée dans le contexte des légendes circulant autour des Sept Sages ${ }^{19}$. Il s'agit donc d'une fiction moralisatrice, dont la dimension paradigmatique est en fait clairement perceptible lorsqu'Hérodote, résumant les propos de Solon à l'occasion de la scène où Crésus monte sur le bûcher, déclare que l'Athénien «ne parlait pas plus pour Crésus que pour tous les hommes, et en particulier pour ceux qui se considéraient être heureux (ỏ $\lambda \beta i ́ o v \varsigma$ )» (I, 86, 5). Le choix du personnage fictif de Solon n'était d'ailleurs pas innocent. On rappellera en effet que, dans ses poèmes, le Solon historique critiquait précisément ceux qui honoraient la richesse jusqu'à

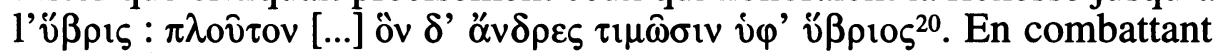
l'üßpıs d'un Crésus qui se croyait «le plus heureux des hommes» grâce à sa fortune, le Solon d'Hérodote s'identifiait donc au personnage historique, si ce n'est qu'à la richesse il substituait une nouvelle conception de l'ô$\lambda \beta o s$, plus morale pourrait-on dire, et non la vertu

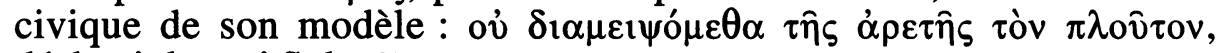
déclarait le vrai Solon ${ }^{21}$.

Finalement, la rencontre fictive entre Solon et Crésus n'avait nullement pour objectif de dénoncer l'insolence d'un Barbare en y opposant une sagesse grecque universelle; l'épisode se voulait davantage une mise en garde contre l'v̈ $\beta \rho 1 s$ dont pouvaient faire preuve les aristocrates grecs. Quant à Crésus, si on ne pouvait ignorer son origine lydienne, c'est d'abord et avant tout son attitude aristocratique qui était au cœur du débat. Pour un Grec de l'époque classique, le roi lydien ne

19 Voir déjà O. Regenbogen, loc. cit. (n. 6), p. 395-398 et, plus récemment, P. Oliva, op. cit. (n. 6), p. 14-17. - Même si l'historien d'Halicarnasse, comme l'a très justement rappelé Tr.S. Brown, loc. cit. (n. 6), ne connaissait pas ce concept des Sept Sages - qui n'apparaît pas dans nos sources avant le IVe siècle -, on admettra volontiers que l'on se trouve ici en face des prémices de cette tradition. Effectivement, di un gruppo di 'Sette sapienti' ci parla espressamente per la prima volta solo Platone, ma molti elementi fanno pensare che il nucleo di questo ciclo di storie (certi personaggi e certo materiale) risalga ad età più antica, fino al VI secolo, écrivait dernièrement A. SANTONI, loc. cit. (n. 15), p. 94.

20 SolON fr. 1, v. 9-13 G.-P. - Sur les réminiscences soloniennes dans le personnage fictif d'Hérodote, il faut consulter l'excellent article de C.C. CHIASSON, loc. cit. (n. 6).

21 SolON fr. 6 G.-P. 
représentait pas tant le souverain oriental qu'il n'était le prototype même de l'aristocrate grec. N'en déplaise à la bibliographie contemporaine, mais l'opposition entre les vertus de la Cité et l'ignorance d'un Barbare ne rencontre pas le moindre point d'ancrage dans le texte.

Dans le second volet de ce dossier athénien, Hérodote raconte comment le roi lydien fit la fortune des Alcméonides. Apprenant qu'Alcméon avait aidé les Lydiens à consulter l'oracle de Delphes, Crésus désira le remercier personnellement. Il le fit venir à Sardes et lui offrit de prendre tout l'or qu'il pouvait emporter sur lui. Alcméon revêtit de larges vêtements, entra dans le trésor et prit tout ce qu'il pouvait, à tel point qu'il en sortit en étant à peine capable de tenir sur ses jambes. Crésus se mit à rire et lui fit cadeau de nombreux présents supplémentaires (VI, 125).

\section{Problème de chronologie}

Tout comme Solon, Alcméon ne semble pas être un contemporain du dernier roi de Lydie. Â l'époque de Crésus, le personnage important de la maison des Alcméonides était Mégaclès, le fils de notre Alcméon, comme l'atteste Hérodote en faisant de celui-ci l'adversaire de Pisistrate (I, 59); ce fils avait d'ailleurs à cette époque une fille nubile qu'il donna en mariage au tyran lors de son premier retour d'exil (I, 60-61). Hérodote rapporte en outre que c'est grâce aux libéralités de Crésus qu'Alcméon avait pu entretenir un attelage et remporter le prix à Olympie (VI, 125,5). On date pourtant cette victoire de 592, ce qui n'a pas de sens par rapport aux années de règne de Crésus (561-547). Dans la VII ${ }^{e}$ Pythique, Pindare, qui dresse un palmarès des succès remportés par les Alcméonides, mentionne en effet une seule victoire à Olympie, qu'une scholie s'empresse de dater de la $47^{\mathrm{e}}$ olympiade ${ }^{22}$. Il faut certes descendre la date traditionnelle de 592 de quelques années, pour retenir

22 Schol. PIND., Pyth. VII Inscr. a Drachmann. - Contrairement à la communis opinio, la scholie ne semble pas distinguer trois Mégaclès : le vainqueur de la course de chars dans la $25^{\mathrm{e}}$ pythiade, un autre, victorieux à Olympie dans la $47^{\mathrm{e}}$ olympiade, et un troisième, remportant le prix dans la $86^{\mathrm{e}}$ olympiade. Le texte grec ne mentionne que deux Mégaclès : le premier et le troisième de la liste. Seul le troisième, en effet, est qualifié de $\tau o v ́ \tau \omega$ ó $\mu \omega ́ v v \mu o \varsigma$, c'est-à-dire homonyme du personnage principal de la notice, le vainqueur célébré par Pindare lors de la $25^{\mathrm{e}}$ pythiade. La scholie ne précise pas le nom du vainqueur de la $47^{\mathrm{e}}$ olympiade et le texte ne permet pas de déduire qu'il s'agissait d'un Mégaclès. En revanche, la seule victoire olympique antérieure à l'ode de Pindare nous est connue par

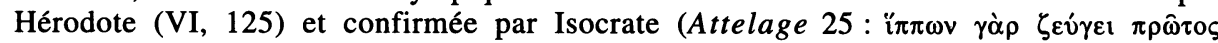

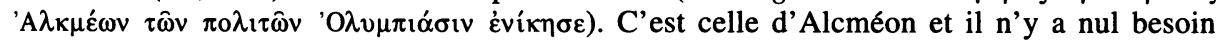
d'imaginer que le scholiaste de Pindare s'est trompé sur son nom. 
celle bien plus correcte de $586^{23}$, mais ce n'est évidemment pas suffisant pour faire d'Alcméon un contemporain de Crésus. Cette date précise permet en revanche d'identifier notre personnage avec l'Alcméon que Plutarque (Solon, 11, 2) mentionne comme stratège des Athéniens lors de la Première Guerre Sacrée.

Dans tous ces textes, Alcméon apparaît donc clairement comme un contemporain d'Alyatte plutôt que de Crésus. Aussi a-t-on parfois voulu remplacer le nom de Crésus par celui de son père qui, lui aussi, envoya une ambassade à Delphes afin de consulter l'oracle ( $c f$. Hérodote, I, 19). On peut notamment lire sous la plume de George Forrest : Alkmeon's visit to Sardis must then be dated to 594 when the war had already settled down to a long dull siege and one of the allied commanders could easily be spared for a goodwill mission to Delphi's most important friend abroad, to assure Alyattes that the new Delphi would be as helpful and co-operative as the old. In 593 he would buy his stable and in 592 was the first (and last) Alkmeonid to win ${ }^{24}$. Cette hypothèse ne me semble pas satisfaisante, car encore faudrait-il expliquer comment le nom de Crésus est venu se substituer à celui d'Alyatte. Peut-être est-il dès lors préférable d'admettre que la rencontre entre Alcméon et Crésus est une fiction, tout comme celle de Solon ${ }^{25}$.

\section{Une interprétation tenace}

C'est dans l'exposé de la première guerre médique que l'on rencontre cet épisode, alors que celui-ci nous renvoie au siècle précédent.

23 Ed. LÉvy, Notes sur la chronologie athénienne au VI siècle. I. Cylon, dans Historia, 27 (1978), p. 513-521, partic. p. 519-520, reprenant la thèse de Th. Lenschau sur la périodicité annuelle - et non pentétérique - des premières olympiades, a rappelé que la $47^{\mathrm{e}}$ olympiade correspondait dès lors à l'année 586. Cette correction des dates olympiques s'applique exclusivement aux vainqueurs qui constituent eux-mêmes la liste, non à toutes les dates traduites en olympiades et antérieures à la $50^{\mathrm{e}}$. Ce sont là deux choses totalement différentes que les historiens confondent trop souvent. - Reprenant la communis opinio ( $c f$. n. 22), Ed. Lévy attribue cette victoire à un Mégaclès : le père d'Alcméon.

24 G. Forrest, The First Sacred War, dans $B C H, 80$ (1956), p. 33-52 (citation, p. 51 ).

25 On a parfois voulu considérer comme un indice de relations réelles entre les Alcméonides et les rois lydiens un kouros découvert à Anavyssos (ATHÈNES, MN 3851) et dont la base (ATHĖNES, MN 4754) porte le nom de Kroisos, car on y voyait un Alcméonide. Voir dernièrement J. MCK. CAMP II, Athens and Attica: The Town and its Contryside, dans A. VerbANCK-PIÉRARD et D. Viviers (éd.), Culture et Cité. L'avènement d'Athènes à l'époque archaïque, Bruxelles, 1995, p. 229 et n. 7 (avec bibliographie). Cette interprétation soulève néanmoins beaucoup trop de problèmes pour être acceptable, $c f$. $\mathrm{D}$. VIVIERS, Recherches sur les ateliers de sculpteurs et la Cité d'Athènes à l'époque archaïque, Bruxelles, 1992, p. 123-124. 
Dans le récit de la bataille de Marathon, Hérodote se fait l'écho d'une rumeur qui aurait couru à Athènes au lendemain du combat. Accusant les Alcméonides, on prétendait qu'un bouclier avait été levé afin d'indiquer aux Perses que la ville était sans défense (VI, 115-116). Quelques pages plus loin, l'historien d'Halicarnasse s'indigne et entame un plaidoyer en faveur des accusés, contestant, point par point, les chefs d'accusation (VI, 121-124) ${ }^{26}$. C'est dans le cadre de ce développement sur les Alcméonides qu'Hérodote rapporte ensuite deux épisodes de leur histoire familiale : d'abord l'aventure d'Alcméon chez Crésus (VI, 125), puis le mariage de son fils Mégaclès avec la fille de Clisthène, le tyran de Sicyone (VI, 126-130).

À l'exception d'un article récent, une interprétation des chapitres 125 à 131 du livre VI domine l'historiographie moderne depuis longtemps. Il s'agit du commentaire souvent cité qu'ont livré W.W. How et J. Wells : H.'s attempt to prove Alcmaeonid hatred of the barbarian and of the tyrant is illogical and unconvincing. Even here (125) he relates their friendship with Croesus, the first barbarian who enslaved Greeks (i. 6. 2), and with Cleisthenes, tyrant of Sicyon (126f.), and he conveniently forgets their alliance with Pisistratus (i. 60; Plut. Mor. 863 $B)^{27}$. Les commentateurs ont en fait repris les «définitions» de Crésus que donne Hérodote en tête de son logos lydien (

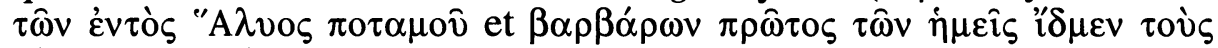

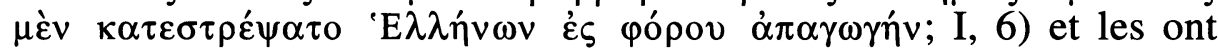
appliquées à la rencontre d'Alcméon. Quant au mariage de Mégaclès, ils n'ont retenu qu'un seul élément : le père de la mariée était un tyran. Les deux épisodes se voyaient donc dotés d'une forte connotation négative en fonction de l'un ou l'autre mot qui, dans le cas de Crésus, n'apparaissait même pas dans le passage. Leur interprétation connut un franc succès dans la bibliographie; R. Develin déclarait à leur suite : It is certainly difficult to suppose that the information here [...] came from a laudatory Alkmeonid tradition ${ }^{28}$.

26 Ce n'est pas ici l'endroit de discuter de la réalité d'une telle accusation. On consultera p.ex. G.M.E. Williams, The Kerameikos Ostraka, dans ZPE, 31 (1978), p. 103113, sur les éclairages intéressants qu'apportent les inscriptions des ostraca du Céramique pour l'étude des cinq années qui suivirent Marathon. Cette analyse lui permet ainsi de rejoindre H.G. HuDSON, The Shield Signal at Marathon, dans AHR, 42 (1937), p. 443-459, et de dire que l'affaire du Marathon shield signal n'avait pas encore été retournée contre les Alcméonides au milieu des années 480, ou même, n'avait pas encore été inventée.

27 W.W. How et J. Wells, A Commentary on Herodotus. Vol. II, Oxford, 1928, p. 115 .

28 R. DEVELIN, Herodotos and the Alkmeonids, dans J.W. EADIE et J. OBER (éds), The Craft of the Ancient Historian. Essays in Honor of C.G. Starr, Lanham, 1985, p. 125-139 (citation, p. 132). - Autre exemple récent, H.Y. McCulloch Jr., Herodotus, Marathon and Athens, dans SO, 57 (1982), p. 35-55: these stories hardly seem to point to a celebration of the Alcmaeonids (p. 45). 
Et pourtant, une telle interprétation des chapitres 125-131 implique très nettement une contradiction interne dans le texte d'Hérodote. Une fois dotés d'une forte connotation négative, l'épisode d'Alcméon et celui du mariage de Mégaclès se placent en effet en opposition directe avec le plaidoyer d'Hérodote dans l'affaire du bouclier de Marathon (121-124). Afin d'éviter cette contradiction, J. Hart écrira d'ailleurs à propos d'Hérodote : his advocacy [...] is a dazzling piece of irony [...] Herodotus did not aim to convince, but to ridicule the 'official' defence put forward for the Alcmeonidae 29 . Au lieu d'invoquer l'ironie de l'auteur ou de remettre en question la cohérence de ces quelques chapitres en fonction d'une certaine conception de Crésus, issue d'un autre passage de l'œuvre, ne faudrait-il pas plutôt retrouver l'image de Crésus qui se dégage de l'épisode d'Alcméon lui-même ? Car si le texte d'Hérodote nous paraît incohérent, c'est peut-être que le personnage de Crésus n'a pas cette valeur négative que lui ont accordée How et Wells ?

Reprenant récemment le problème, Gabriele Bockisch a très heureusement rompu avec l'interprétation traditionnelle, en proposant une réhabilitation des Alcméonides et de leurs contacts avec le roi lydien. Elle considérait l'épisode comme der erste Beweis für Perser- und Tyrannenfeindlichkeit ${ }^{30}$. Selon cette interprétation, Crésus ne symboliserait en effet pas tant le tyran barbare qu'il n'incarnerait le premier adversaire des Perses. Par conséquent, en prétendant avoir aidé le roi lydien à consulter l'oracle de Delphes, les Alcméonides auraient en fait témoigné leur haine à l'égard des Perses, non leur soutien à un tyran barbare. Disons-le d'emblée, cette analyse est salutaire, car elle permet de rendre sa cohérence au texte de l'historien : désormais favorable aux Alcméonides, l'épisode s'insère sans plus aucune difficulté à la suite du plaidoyer passionné d'Hérodote contre une accusation qui faisait d'eux des philobarbaroi. Pourtant, rien dans l'épisode ne présente Crésus comme l'adversaire des Perses et, si Hérodote évoque effectivement l'oracle de Delphes, il faut en revenir au logos de Crésus (I, 46-56) pour comprendre dans quel contexte se situait cette consultation. On se retrouve donc finalement dans une situation similaire à celle de How et Wells : l'interprétation du récit en fonction d'une conception de Crésus étrangère à l'épisode lui-même. La question de la connotation attachée au dernier roi lydien dans cet épisode-ci reste donc entièrement posée.

29 J. HART, Herodotus and Greek History, Londres, 1982, p. 12.

30 G. BoCKISCH, Alkmeon bei Kroisos, dans $A C D, 26$ (1990), p. 9-17 (citation, p. 16). 


\section{Problème de critique interne}

Quel pouvait être l'objectif d'Hérodote lorsqu'il rapportait l'histoire d'Alcméon à Sardes et celle du mariage de Mégaclès? Il

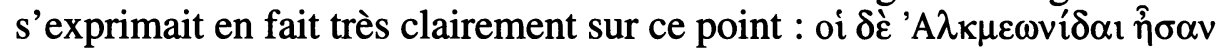

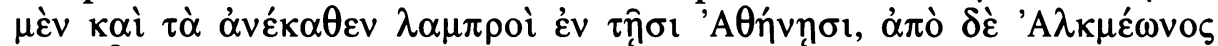

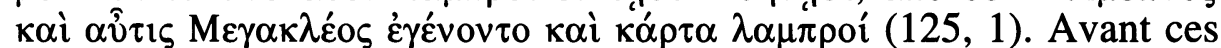
deux personnages, les Alcméonides étaient certes illustres à Athènes $(\lambda \alpha \mu \pi \rho o i)$, mais c'est grâce à eux qu'ils le devinrent tout à fait (кó $\rho \tau \alpha$

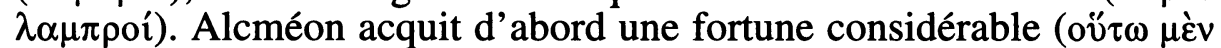

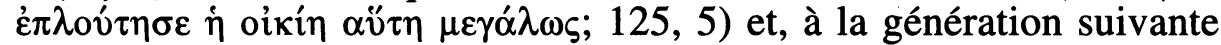

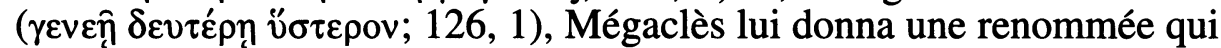

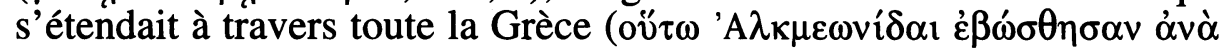
$\tau \eta ̀ v ~ ' E \lambda \lambda \alpha ́ \alpha \alpha ; 131,1)$, en étant choisi par Clisthène de Sicyone comme le

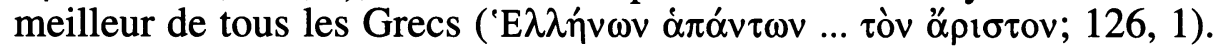
Hérodote a établi entre les deux épisodes un lien réel, un balancement constant de l'un à l'autre. Il forma ainsi un ensemble cohérent de deux histoires qui, à l'origine, n'avaient sans doute rien en commun, sinon qu'elles célébraient toutes deux la gloire des Alcméonides. Mais quel rapport y a-t-il entre cet ensemble de deux histoires (125-130) et le plaidoyer qu'il prononce juste auparavant (121-124)?

Il semble en fait qu'il n'y en ait aucun. Le plaidoyer (121-124) a pour but de laver les Alcméonides de tout soupçon dans l'affaire du bouclier de Marathon, tandis que l'auteur cherche par les deux histoires suivantes (125-130) à montrer comment les Alcméonides ont acquis une renommée s'étendant à toute la Grèce. Le thème est totalement différent et il n'y a aucune raison de penser que les deux anecdotes constituent la suite logique de son discours. D'autant qu'Hérodote a terminé son plaidoyer avant de passer à l'apologie des Alcméonides; tous les arguments ont été évoqués. "Comment osait-on porter un tel grief à l'encontre de ceux qui haïssaient à ce point les tyrans qu'ils vécurent en exil tant qu'Athènes était sous leur joug, eux qui, plus qu'Harmodios et Aristogiton, en furent les libérateurs (121-123) ?» «Pourrait-on même les accuser de vouloir trahir leur patrie en la livrant aux Barbares, alors qu'ils avaient reçu tant d'honneurs des Athéniens (124) ?» N'ayant pas d'autre élément à ajouter, Hérodote clôt d'ailleurs son plaidoyer en revenant sur un plan plus strictement événementiel : nul doute pour lui

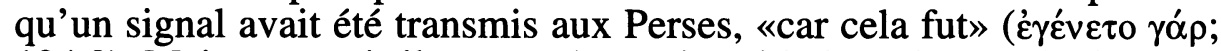
124,2). Mais, poursuit-il, «quant à savoir qui le leva, je ne sais rien dire

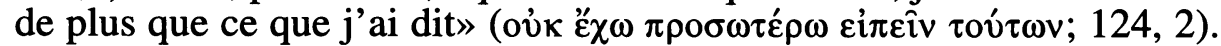
La discussion est bel et bien close. Finalement, ni une parenté de thème, ni une continuité logique ne permet d'établir un lien direct entre le plaidoyer en faveur des Alcméonides (121-124) et leur apologie (125- 
130). Ajoutons qu'il faut en outre distinguer les deux niveaux de discours qui se succèdent au cours des chapitres 121-131. La défense est

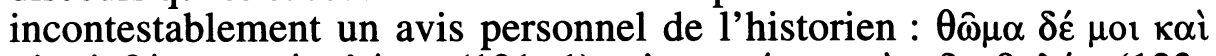

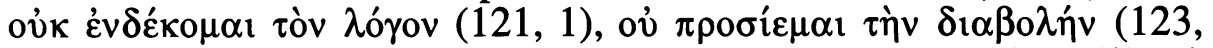

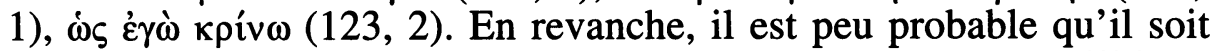
l'inventeur des récits relatifs à Alcméon et à Mégaclès, même s'il les a réunis en un thème commun, la renommée des Alcméonides.

Hérodote a donc simplement profité de l'occasion que lui fournissait l'affaire du bouclier de Marathon pour introduire deux histoires élogieuses qu'il avait entendues au sujet de cette famille. Dès lors, si l'interprétation négative de How et Wells doit être rejetée parce qu'elle rend le texte de l'historien incohérent, la thèse de G. Bockisch pêche sans doute par excès de cohérence : la venue d'Alcméon à Sardes n'est pas der erste Beweis für Perser- und Tyrannenfeindlichkeit. Quel sens lui donner dès lors?

\section{La générosité de Crésus}

Comme on l'a vu, Hérodote utilise l'histoire d'Alcméon afin de faire l'apologie des Alcméonides. Il cherche à montrer à son lecteur comment cette famille avait acquis sa richesse (oü $\tau \omega \mu \dot{\varepsilon} v \dot{\varepsilon} \pi \lambda$ ov́ $\tau \sigma \sigma \dot{\eta}$

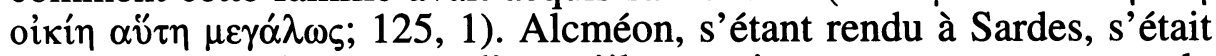
vu offrir par Crésus autant d'or qu'il pourrait en emporter en une seule fois. L'Athénien enfila les vêtements les plus larges qu'il trouva, entra dans le trésor et se chargea de tout l'or qu'il put prendre sur lui. À sa sortie, il ressemblait à tout sauf à un homme et le roi lydien se mit à rire.

Bien sûr, l'attitude d'Alcméon dans le trésor de Crésus n'est pas des plus nobles; bien sûr, il fait rire le roi lydien ${ }^{31}$. Mais il faut bien reconnaître qu'Alcméon ne faisait que répondre à une invitation insolite de Crésus. Hérodote est tout à fait explicite sur ce point : ó $\delta \dot{\varepsilon}$ 'A $A \kappa \mu \varepsilon ́ \omega v$

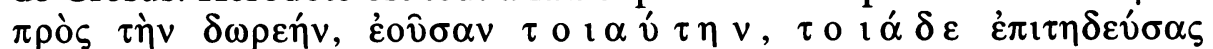
$\pi \rho о \sigma \varepsilon ́ \varphi \varepsilon \rho \varepsilon(125,3)$, ce que je traduirais volontiers par devant un présent aussi inhabituel Alcméon se comporta de la même manière. Quelle que

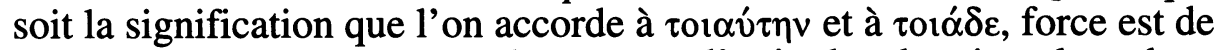
constater qu'il y a correspondance entre l'attitude adoptée et le cadeau offert : si le comportement d'Alcméon fut tel, c'est parce que les paroles de Crésus l'étaient aussi. Alcméon n'est pas ridicule, il s'est montré plus rusé que le roi lydien; et si ce dernier rit, ce n'est pas pour se moquer de l'Athénien, mais parce qu'il est étonné d'avoir été pris à son propre jeu.

$31 C f$. W.W. How et J. WELls, op. cit. (n. 27), p. 116 : the story [...] is obviously a comic version of the enrichment of the family. 
C'est la ruse qui permet à Alcméon d'emporter tant d'or, au point que le roi le récompense par des présents supplémentaires.

Pourtant, on oppose bien souvent l'indifférence de Solon face aux trésors de Crésus à l'avidité d'Alcméon dans la même situation, et cela afin de renforcer l'aspect négatif que l'on accorde traditionnellement à cette histoire $^{32}$. Les comparaisons ne constituent peut-être pas la meilleure méthode pour découvrir la valeur et la fonction de deux récits, dont l'origine est manifestement différente. En l'occurrence, on rappellera que Solon ne critique jamais la richesse de Crésus; c'est l'v̋ßpı que provoquent sa thésaurisation et son ostentation qu'il réprouve. Or, c'est précisément la générosité du roi lydien qu'illustre ici la rencontre avec Alcméon. D'ailleurs, si l'on poursuit les comparaisons, l'or que reçoit Alcméon n'est fondamentalement pas différent de celui que Delphes exhibe. Tout le monde a en tête la description des fabuleuses offrandes de Crésus ( $c f$. Hérodote, I, 50-51) et, connaissant les liens qui unissaient le sanctuaire et les Alcméonides, il ne serait pas raisonnable de penser que l'or d'Alcméon n'avait pas la même valeur morale que celui de Delphes. Dans tous les cas, l'or de Crésus n'était autre que de l'«argent propre», si bien qu'en inventant ce récit, les Alcméonides voulaient en fait accroître la réputation de leur fortune, en la faisant dériver, tout comme celle du sanctuaire de Delphes, de la «générosité inoubliable» du richissime Crésus. Que l'on se rappelle en effet les mots de

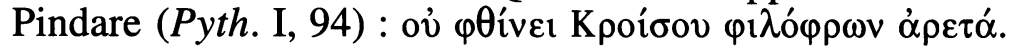

\section{Les Alcméonides à Delphes}

D'autre part, compte tenu de la finalité de ce récit fictif, s'il fallait le rattacher à une accusation portée contre les Alcméonides, plutôt qu'à l'affaire du bouclier de Marathon, ne pourrait-on pas songer à l'accusation d'avoir détourné les fonds qui leur avaient été confiés pour la reconstruction du temple de Delphes ? Revenons en effet brièvement sur cette affaire.

Tandis qu'Hérodote $(\mathrm{V}, 62)$ et Pindare (Pyth. VII) se contentent de louer les Alcméonides, qui non seulement reconstruisirent le temple détruit en 548/7, mais encore en édifièrent la façade en marbre de Paros (ce qui n'était pas prévu dans le cahier des charges), on sait qu'au IVe siècle, plusieurs auteurs ${ }^{33}$ prétendirent que la reconstruction du temple n'avait été, pour les Alcméonides, qu'un moyen d'obtenir de l'argent

32 P. ex. H.Y. McCulloch Jr., loc. cit. (n. 28), p. 45 : Herodotus certainly must have wanted his readers to compare Alcmaeon's immoderate behavior to Solon's sober behavior in that same treasury.

33 Philochore, 328 F 115 J.; Aristote, Const. Ath., 19, 4; DÉmosthène, 21 (Contre Midias), 144; IsOCRATE, 15 (Sur l'échange), 232. 
afin d'acheter l'aide des Spartiates pour expulser les tyrans d'Athènes. La bibliographie s'est longtemps intéressée au déroulement des événements, ce qui l'amena à critiquer les lacunes du récit d'Hérodote pour suivre la tradition atthidographique ${ }^{34}$. Dernièrement pourtant, $M$. Stahl a insisté sur la signification hautement symbolique de cette entreprise delphique pour les Alcméonides, la tenant pour un exemple d'émulation aristocratique et rendant ainsi du crédit au texte d'Hérodote ${ }^{35}$. Si l'on va plus loin, ne pourrait-on pas également penser que nous nous trouvons ici en présence de deux versions partisanes des événements : d'une part, chez les Atthidographes, l'accusation - fondée ou non, peu importe ici de s'être servi des fonds alloués à d'autres fins que la construction du temple, d'autre part, chez Hérodote, la défense des Alcméonides, qui faisaient valoir non seulement qu'ils étaient riches et célèbres depuis

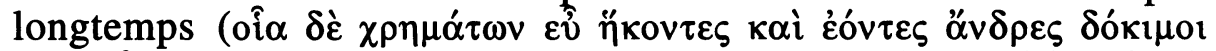

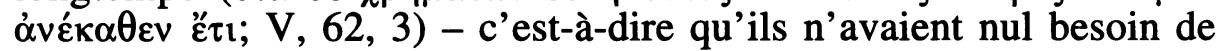
l'argent de Delphes pour chasser les tyrans -, mais encore qu'ils avaient même dépensé plus que prévu en construisant une façade en marbre de Paros ? On reconstituerait ainsi volontiers une part des débats auxquels donna lieu cette controverse delphique.

Si l'on veut bien admettre ce qui précède, on en trouvera peut-être un indice supplémentaire dans la visite d'Alcméon à Sardes. On ne peut en effet qu'être frappé par le rappochement d'un épisode qui visait précisément à établir l'ancienneté de la richesse des Alcméonides ( $c f$. $\dot{\alpha} v \varepsilon ́ \kappa \alpha \theta \varepsilon v \lambda \alpha \mu \pi \rho o i ́ ;$ VI, 125,1$)$ avec la défense que ceux-ci développèrent dans le cadre de l'affaire delphique. Dans les deux cas, l'argumentation est identique. Grâce aux dons de Crésus, les Alcméonides faisaient non seulement remonter leur richesse bien au-delà de l'adjudication des travaux du temple de Delphes, mais encore cette fortune était blanchie par une origine tout à fait prestigieuse, dont Delphes elle-même se glorifiait en exhibant les offrandes de Crésus. Finalement, loin d'être une attaque de «philobarbarie» dirigée contre les Alcméonides, la rencontre fictive de Crésus et d'Alcméon aurait parfaitement pu les défendre face à une rumeur de détournement de fonds. Quant à Crésus, c'est une nouvelle fois sa richesse et le prestige aristocratique de celle-ci qui en constituent les traits les plus significatifs.

\footnotetext{
34 Voir p. ex. F. JACOBY, FGrHist III b Suppl., p. 453-454.

35 Die Rolle der Alkmeoniden in Delphi bestand also darin, freiwillig und aus eigenen Mitteln etwas zum Bau des Apollon-Tempels [...] beizutragen, nicht weil sie speziell dafür qualifiziert waren, sondern weil es die ihnen als Aristokraten obliegende Verplichtung so forderte, déclarait-il. - M. STAHL, Aristokraten und Tyrannen im archaischen Athen, Stuttgart, 1987, p. 120-133 (citation, p. 130).
} 
Venons-en au dernier volet de ce dossier athénien. Dans son récit de la colonisation athénienne en Chersonèse, Hérodote rapporte que Miltiade l'Ancien fut capturé par ses ennemis lors d'une guerre contre Lampsaque. L'œciste fit appel à Crésus, avec qui il entretenait de bonnes relations; ce dernier menaça les Lampsacéniens d'extermination s'ils ne relâchaient pas Miltiade (VI, 37). Comme pour l'épisode d'Alcméon, c'est l'Athénien qui focalisa l'attention des commentateurs, tandis que le personnage de Crésus fut à nouveau interprété en fonction d'une idée préconçue.

\section{L'histoire philaïde dans l'œuvre d'Hérodote}

Les Philaïdes ne sont que des acteurs parmi d'autres dans l'œuvre d'Hérodote. Il n'y a pas, comme pour les Alcméonides (VI, 121-131), de passage où l'auteur se concentre exclusivement sur cette famille. Les données relatives à Miltiade l'Ancien et à son neveu, Miltiade le Jeune, sont éparses dans les Histoires : du livre IV, où il est question de l'expédition de Darius en Scythie - à laquelle participa Miltiade le Jeune -, au livre VI et au récit de la bataille de Marathon. Bien que disséminée à travers des passages totalement différents, l'histoire philaïde chez Hérodote n'en est pas moins unitaire. On a en effet montré qu'une partie des traditions rapportées par Hérodote s'organisait en fonction des deux procès de Miltiade le Jeune ${ }^{36}$. Le premier, en 493, frappa l'Athénien à son retour de Chersonèse; on l'accusa d'y avoir exercé la tyrannie. Le second, en 489, après l'échec de l'expédition de Paros, fut intenté par Xanthippos, qui le traîna en justice pour avoir trompé le peuple. Dans les deux cas, des arguments furent développés par l'accusation et par la défense, mais seul le second procès fit l'objet d'un compte rendu détaillé d'Hérodote $^{37}$. Afin de reconstituer les débats que suscita le premier procès, il s'agit donc de vérifier si les données du récit d'Hérodote sur les Philaïdes en Chersonèse, ou au moins certaines d'entre elles, $s$ 'insèrent volontiers dans le contexte du premier procès de Miltiade ${ }^{38}$. Effectivement, une grande partie des traditions rapportées par Hérodote présentent les Philaïdes tantôt comme des adversaires des tyrans athéniens - le meurtre du père de Miltiade le Jeune, Cimon Coalémos, par les

36 R. CRAHAY, La littérature oraculaire chez Hérodote, Paris, 1956, p. 261-272; D. VIVIERS, Historiographie et propagande politique au $V^{e}$ siècle avant notre ère: les Philä̈des et la Chersonèse de Thrace, dans RFIC, 115 (1987), p. 288-313.

37 HÉRODOTE, VI, 136 : face à l'accusation de Xanthippos, la défense fit valoir le comportement de Miltiade dans la bataille de Marathon (VI, 102-116) et rappela la prise de Lemnos (VI, 137-140). - Le fait que le premier procès ne donne pas lieu à un exposé aussi clair des arguments a même conduit certains, comme K.J: Beloch, à mettre en doute l'historicité de ce premier procès. Contra D. VIVIERS, loc. cit. (n. 36), p. 292, n. 5.

38 D. VIVIERS, loc. cit. (n. 36), p. 292. 
fils de Pisistrate (VI, 103) -, tantôt comme des tyrans eux-mêmes - la prise de pouvoir autoritaire, comparable à celle de Pisistrate, par laquelle Miltiade le Jeune reprit en mains les affaires de Chersonèse à la mort de son frère Stésagoras (VI, 39). Comme le procès de 493 portait sur une accusation de tyrannie, on est donc tenté de voir en ces traditions des arguments développés tantôt par la défense tantôt par l'accusation. En est-il de même pour l'intervention de Crésus dans la guerre contre Lampsaque, et de quel côté faut-il placer ce récit? En l'absence de précision de la part d'Hérodote, la voie était libre à toutes les interprétations.

Crésus, le «tyran par excellence»

C'est Roland Crahay qui donna le ton, en partant précisément du personnage de Crésus : Hérodote rapporte différents faits qui ont dû être mis en avant par les accusateurs : l'anecdote où Crésus, le $\tau$ v́ $\rho \alpha v$ v $\varsigma$ par excelle $n c e$, protège Miltiade l'Eciste contre les Lampsacéniens, le fait que Miltiade II aurait obtenu une trirème des Pisistratides pour aller prendre possession de son héritage [...] le coup de force de Miltiade II contre les notables Chersonésiens ${ }^{39}$. Une interprétation semblable, se fondant également sur une conception préétablie de Crésus, fut à nouveau avancée par H.Y. McCulloch, au départ d'un rapprochement avec l'épisode d'Alcméon : the association between Alcmaeon and Crosus is much the same as that between Miltiades' uncle, also named Miltiades, and Crosus (6.37). Both the Philaids and the Alcmaeonids were tainted by this connection ${ }^{40}$. En dernier lieu, Didier Viviers ne prit pas position pour ou contre Crésus, mais rappela que certains éléments des chapitres VI, 37-38 (circonstances des guerres contre Lampsaque et organisation de jeux en l'honneur de Miltiade l'Ancien) pouvaient avoir été recueillis par Hérodote en Chersonèse même ${ }^{41}$. Cela n'interdit toutefois nullement $d^{\prime} y$ entrevoir également une utilisation politique dans le cadre du procès de 493, au-delà du topos Crésus-tyran.

Lampsaque, la cité des tyrans

C'est au cours d'une guerre contre Lampsaque que Miltiade l'Ancien fut fait prisonnier; et la cité ennemie n'est peut-être pas sans importance, car l'hostilité des Philaïdes envers les Lampsacéniens est

39 R. CRAHAY, op. cit. (n. 36), p. 265 (c'est moi qui espace).

40 H.Y. McCulloch, loc. cit. (n. 28), p. 45. La référence à la lecture de l'épisode d'Alcméon (VI, 125) par How et Wells est évidente !

41 D. VIVIERS, loc. cit. (n. 36), p. 292, n. 3. 
bien attestée dans l'ensemble de la tradition. En effet, le neveu et successeur de Miltiade l'Ancien à la tête de la Chersonèse, Stésagoras, trouva la mort lors d'une nouvelle guerre contre Lampsaque (VI, 38). On sait également que les Chersonésitains rendaient un culte à l'œciste et qu'ils célébraient des jeux hippiques et gymniques en son honneur, mais aucun Lampsacénien n'avait le droit d'y participer (VI, 37-38). En outre, Hippoclos de Lampsaque avait ouvertement pris le parti opposé à Miltiade le Jeune lors de la discussion entre les Grecs qui accompagnaient Darius en Scythie. Miltiade y avait exposé un avis enjoignant ses collègues à abandonner le Grand Roi de l'autre côté du Danube et à libérer les cités d'Ionie (IV, 136-138). Les sources montrent donc, avec une insistance toute particulière, une véritable opposition héréditaire des Philaïdes aux Lampsacéniens.

Or, les liens très étioits que cette cité entretenait avec les tyrans athéniens étaient bien connus : le fils d'Hippoclos, le tyran de Lampsaque, avait épousé la fille d'Hippias; la stèle funéraire de celle-ci s'y trouvait et elle s'y vantait d'avoir été fille, épouse, sœur et mère de tyrans. C'est encore à Lampsaque qu'Hippias se retira une fois expulsé d'Athènes, avant de rejoindre Darius ${ }^{42}$. D'autre part, D. Viviers a montré que le conseil tenu sur les rives du Danube s'insérait vraisemblablement dans le contexte du premier procès de Miltiade le Jeune ${ }^{43}$. La forte connotation tyrannique associée au nom de Lampsaque (tant par son régime que par les liens qui l'unissaient aux Pisistratides) ainsi que l'actualité d'une opposition au tyran Hippoclos dans la défense prononcée en 493 conduisent à penser que cet épisode d'une guerre entre Miltiade l'Ancien et Lampsaque pourrait également faire partie de la propagande développée par les Philaïdes au retour de Miltiade le Jeune en 493. Bien que cette guerre fût perdue - tout comme celle où Stésagoras trouva la mort -, et fût en soi peu valorisante, elle permettait au moins aux Philaïdes de démontrer leur opposition intrinsèque à la tyrannie, tant athénienne que lampsacénienne.

\section{Le rôle de Crésus}

Si la lutte contre Lampsaque relevait effectivement de la propagande philaïde et non de l'accusation, comme le pensaient Crahay et McCulloch, comment faut-il dès lors comprendre l'intervention de Crésus en faveur de Miltiade l'Ancien ? Si l'on considère le roi lydien comme un tyran (comme pourrait nous y conduire l'introduction du logos de Crésus), il faut bien reconnaître que Lampsaque était tout autant, si pas plus, liée à la tyrannie. Qui plus est, seule cette dernière

\footnotetext{
42 THUCYDIDE, VI, 59.

43 D. Viviers, loc. cit. (n. 36), p. 295.
} 
entretenait des relations suivies avec les tyrans athéniens - ce qui ne devait pas manquer d'être une image forte dans l'Athènes des années 490 -, alors qu'Hérodote nous dit que Crésus avait refusé de s'allier à

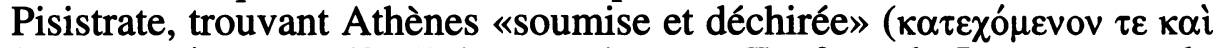
$\delta 1 \varepsilon \sigma \pi \alpha \sigma \mu \varepsilon ́ v o v ;$ I, 59, 1) à cette époque. En face de Lampsaque, le personnage de Crésus ne devait donc revêtir aux oreilles d'un auditoire athénien aucune connotation particulièrement négative et son évocation ne porter aucun préjudice aux Philaïdes; bien au contraire.

L'intervention du roi lydien en faveur de Miltiade l'Ancien était due, nous dit Hérodote, aux bonnes relations que l'œciste entretenait

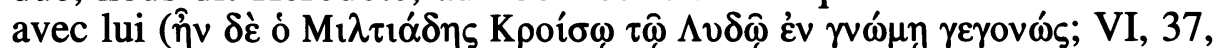
$1)^{44}$. Certes, aucun élément ne vient préciser la nature des relations qui unissaient Miltiade au roi lydien; mais, à la différence d'autres rencontres, aucune incompatibilité chronologique ne fait obstacle à ce récit $^{45}$ et, s'il fut effectivement utilisé par la propagande philaïde, il n'en contient peut-être pas moins des éléments historiques ${ }^{46}$. L'évocation de ces liens s'inscrivait par ailleurs parfaitement dans une mentalité aristocratique, où les relations avec les grands personnages contribuaient fortement au prestige d'une famille ${ }^{47}$. Et le roi lydien était sans doute le plus illustre personnage avec qui on pouvait entrer en contact. Peut-être d'ailleurs y avait-il une volonté de la part des Philaïdes de rivaliser avec les Alcméonides, qui eux aussi prétendaient avoir connu Crésus. Finalement, pas plus dans le cas d'Alcméon que dans celui de Miltiade, le Lydien ne représentait «le tyran par excellence». Il était bien «l'aristocrate par excellence».

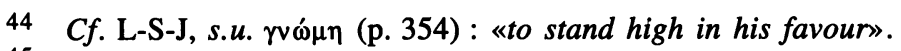

45 La chronologie de Miltiade l'Ancien est assez floue. Elle repose essentiellement sur deux éléments : son départ d'Athènes alors que Pisistrate était au pouvoir (VI, 35) et ses relations avec Crésus (VI, 37). Sans entrer dans le problème de la chronologie de la tyrannie athénienne, disons que l'envoi de Miltiade en Chersonèse ne peut avoir eu lieu qu'à deux moments : entre $561 / 0$ et $556 / 5$, ou après 546/5. Dans la seconde hypothèse, toute rencontre avec le roi lydien, vaincu en 547/6, serait impossible. On s'accorde donc à penser que le départ de Miltiade se fit avant 556/5. - Cf. p.ex. H. BERve, Miltiades. Studien zur Geschichte des Mannes und seiner Zeit, Berlin, 1937 (Hermes Einzelschriften, 2), p. 8.

46 On pense notamment à l'accès au monde thrace, si riche en mines d'or, que pouvaient procurer à Crésus des contacts avec Miltiade l'Ancien en Chersonèse. On sait les Lydiens influents en Bithynie, en Troade et en Propontide ( $c f$. STRABON, XIII, 1, 22; XIII,

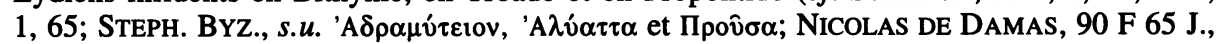
HÉrodote, I, 28), tandis que Miltiade le Jeune épousa la fille du roi des Thraces (HÉRODOTE, VI, 39) et que Darius, plus tard, ordonna à Mégabaze de conquérir ce pays $(\mathrm{V}, 2)$ [sur l'importance de la Thrace dans l'expédition de Scythie menée par Darius, voir dernièrement P. BRIANT, Histoire de l'Empire perse de Cyrus à Alexandre, Paris, 1996, p. 154-158]. Die energische Intervention des Kroisos zu Gunsten des Miltiades [...] ist m. E. aus mehreren Gründen zu erklären, déclarait d'ailleurs Chr. DANOv, Altthrakien, Berlin - New York, 1976, p. 245 .

47 Voir p. ex. l'étude de M. Stahl, op. cit. (n. 35), passim. 
Dans la bibliographie relative aux trois récits examinés ici, le personnage de Crésus a toujours été perçu comme un tyran oriental, dont la morale était bien éloignée des valeurs du citoyen grec et dont la fréquentation était peu recommandable. Effectivement, ces épisodes ont toujours été interprétés en fonction d'une image empruntée à un passage d'Hérodote $(I, 6)$ où l'auteur dresse un portrait certes explicite du dernier roi lydien, mais qui correspond en fait à un tout autre contexte. En introduisant le logos de Crésus, Hérodote désirait en effet présenter le dernier Mermnade comme un tyran barbare soumettant à sa puissance l'ensemble des Grecs d'Asie Mineure, afin de pouvoir l'insérer au vaste projet qu'il venait de formuler : décrire les origines du conflit entre les

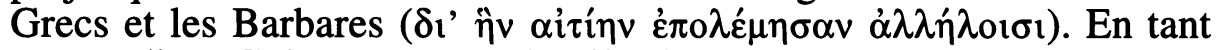
que Lydien, Crésus ne pouvait effectivement se ranger, dans l'esprit d'Hérodote, que du côté des Barbares; ce qui explique qu'il en ait orienté la présentation dans un sens bien précis. Ce n'est cependant pas cette image purement hérodotéenne que présentent les trois récits étudiés ici, dont la conception et l'utilisation idéologique ou politique sont antérieurs, comme on l'a vu, au travail de l'historien d'Halicarnasse. Si un aspect du personnage de Crésus émerge de ceux-ci, c'est d'abord et avant tout sa richesse. Celle-ci est certes tantôt valorisée (lorsqu'elle permet de louer la générosité du roi lydien) tantôt dévalorisée (lorsqu'on critique sa thésaurisation jusqu'à l'ứ $\beta$ pls), mais, dans tous les cas, elle exprime l'identité aristocratique profonde du personnage. C'est dire, finalement, qu'aucun caractère spécifiquement «barbare» ne semble avoir été associé, dans l'Athènes du Ve siècle, à l'évocation du roi lydien.

Enfin, si à l'époque classique, il y eut constitution d'une image cohérente, il y eut surtout instrumentalisation de la figure de Crésus, en ce sens que les Athéniens se souciaient peu de relater des faits réels ou de dresser, en toute objectivité, le portrait du dernier roi de Lydie tel qu'il vécut au VIe siècle. Crésus était devenu l'instrument de l'idéologie aristocratique athénienne. Dans la fiction «solonienne», le jugement négatif porté à l'encontre de l'attitude du roi lydien se voulait une critique du comportement arrogant de certains aristocrates athéniens, afin de leur faire adopter une position plus morale envers les biens matériels. L'aventure d'Alcméon fut, elle, inventée et exploitée par les Alcméonides, leur permettant ainsi d'accroître le prestige de leur fortune, voire d'en blanchir l'origine quelque peu douteuse. Quant à l'épisode (sans doute véridique) de Miltiade l'Ancien, son exploitation permettait non seulement de rappeler l'opposition intrinsèque des Philaïdes à la tyrannie, mais également, grâce à l'intervention du puissant roi de Lydie, de mettre en valeur les contacts internationaux prestigieux qu'entretenait cette famille. En devenant très tôt un personnage stéréotypé, souvent mis en scène dans des histoires fictives, Crésus, à l'instar de Solon, avait en 
somme acquis la force d'un symbole, pour ne pas dire le statut d'une figure mythique.

Rue Saint-Antoine, 46 Alain DUPLOUY

B-5651 Somzée 